24th September 2019

BARI-TH/00

\title{
Measuring the Relativistic Perigee Advance with Satellite Laser Ranging
}

\author{
Lorenzo Iorio $^{\dagger}$, Ignazio Ciufolini ${ }^{\ddagger}$, Erricos C. Pavlis* \\ †Dipartimento di Fisica dell' Università di Bari, via Amendola 173, 70126, Bari, Italy \\ $\ddagger$ Dipartimento di Ingegneria dell'Innovazione dell'Università di Lecce, via per Arnesano, \\ 73100, Lecce, Italy \\ * Joint Center for Earth Systems Technology, JCET/UMBC and NASA Goddard Space \\ Flight Center, Greenbelt, Maryland, 20771-0001 U.S.A.
}

\begin{abstract}
The pericenter advance of a test body by a central mass is one of the classical tests of General Relativity. To day this effect is measured with radar ranging by the perihelion shift of Mercury and other planets, in the gravitational field of Sun, with a relative accuracy of the order of $10^{-2}-10^{-3}$. In this paper we explore the possibility of a measurement of the pericenter advance in the gravitational field of Earth by analyzing the laser-ranged data of some orbiting, or proposed, laser-ranged geodetic satellites. Such a measurement of the perigee advance would place limits on hypothetical, very weak, Yukawa-type components of the gravitational interaction with a finite range of the order of $10^{4} \mathrm{~km}$. Thus, we show that, at the present level of knowledge of the orbital perturbations, the relative accuracy, achievable with suitably combined orbital elements of LAGEOS and LAGEOS II, is of the order of $10^{-3}$. With the corresponding measured value of $\frac{2+2 \gamma-\beta}{3}$, by using $\eta=4 \beta-\gamma-3$ from Lunar Laser Ranging, we could get an estimate of the PPN parameters $\gamma$ and $\beta$ with an accuracy of the order of $10^{-2}-10^{-3}$. Nevertheless, these accuracies would be substantially improved in the near future with the new Earth gravity field models by the CHAMP and GRACE missions. The use of the perigee of LARES (LAser RElativity Satellite), with a suitable combination of orbital residuals including also the node and the perigee of LAGEOS II, would also further improve the accuracy of the proposed measurement.
\end{abstract}




\section{Introduction}

The well known secular shift of the pericenter $\omega$ of a test body induced by the Schwarzschild metric generated by a static, spherically symmetric distribution of mass [Misner et al., 1973; Ciufolini and Wheeler, 1995] is one of the classical tests of Einstein's General Relativity.

\subsection{The Solar System tests}

The advance of pericenter, which can be expressed in terms of $\frac{2+2 \gamma-\beta}{3}$, where $\beta$ and $\gamma$ are the Eddington-Robertson-Schiff PPN parameters [Will, 1993], has been measured in the Solar System, on the Mercury perihelion, by radar signals transmitted from Earth to Mercury and back to Earth [Shapiro et al., 1972], yielding $\frac{2+2 \gamma-\beta}{3}=1.005 \pm 7 \times 10^{-3}$. The contribution of possible systematic errors raises the uncertainty to about $2 \times 10^{-2}$. From the analysis of ten years of data the relative error published in [Shapiro et al., 1976] amounts to $5 \times 10^{-3}$. The major sources of systematic error in this measurement are the poorly-known variations in the topography of the planet's surface and the uncertainties in the radar scattering law [Shapiro, 1990; Pitjeva, 1993]. For a review of periastron advance measurements on binary pulsars see [Will, 2001] and references therein.

Several space missions have been proposed in order to measure, among other effects, this phenomenon by using a variety of techniques: the most recent and promising are SORT, IPLR and ASTROD [Ni, 2001].

More accurate measurements of $\frac{2+2 \gamma-\beta}{3}$ might be performed in the future by means of the ESA BepiColombo Mercury orbiter [Balogh et al., 2000; Milani et al., 2001a; Milani et al., 2001b]. In [Milani et al., 2001b] it is claimed that the BepiColombo mission, which should be launched in 2009, should allow to measure $\gamma$ and $\beta$ with a relative accuracy of the order of $2 \times 10^{-6}$.

\subsection{The proposed experiment}

In this paper we propose to perform a measurement of the general relativistic "gravitoelectric" pericenter advance in the gravitational field of the Earth by using some suitable combinations of the orbital residuals of existing or proposed, spherical, passive, geodetic laser-ranged satellites 
[Iorio, 2002a] with particular emphasis to LAGEOS and LAGEOS II in order to exploit the relevant experience obtained with the gravitomagnetic Lense-Thirring measurements in [Ciufolini et al., 1996; 1997; 1998]. In [Ciufolini and Matzner, 1992] a determination of the LAGEOS general relativistic gravitoelectric perigee shift in the field of the Earth is reported, but the accuracy amounts only to $2 \times 10^{-1}$.

This paper is organized as follows: in section 2 we compare the present experimental accuracy in satellite laser-ranging measurements with the relativistic expression of the perigee shift of the two LAGEOS satellites. In section 3 we analyze some of the most important sources of systematic errors. The accuracy achievable in measuring $\beta$ and $\gamma$ is also discussed. By detecting the relativistic perigee rate of the proposed LARES [Ciufolini, 1998] laser-ranged satellite, in conjunction with the node and the perigee of LAGEOS II, it might be possible to further improve the accuracy in the measurement of these parameters over a long enough time span. This is the subject of section 4 together with an analysis of the potential role of the existing laser-ranged satellite Starlette. Section 5 is devoted to the conclusions.

\section{The relativistic perigee precession of LAGEOS type satellites}

As known, in the slow-motion and weak-field approximation, the Schwarzschild metric generated by a static, spherically symmetric distribution of mass-energy induces an additional post-Newtonian "gravitoelectric force" which acts on the orbit of a test body by shifting its pericenter; in the PPN formalism the pericenter rate can be written as:

$$
\dot{\omega}_{\mathrm{GE}}=\frac{3 n G M}{c^{2} a\left(1-e^{2}\right)} \times \frac{2+2 \gamma-\beta}{3},
$$

in which $G$ is the Newtonian gravitational constant, $c$ is the speed of light in vacuum, $M$ is the mass of the central object, $a$ and $e$ are semimajor axis and eccentricity, respectively, of the orbit

of the test body and $n=\sqrt{G M / a^{3}}$ is its mean motion. In the following we define $\nu \equiv \frac{2+2 \gamma-\beta}{3}$. General Relativity predicts that the perigee shifts for LAGEOS and LAGEOS II amount to 3,312.35 milliarcseconds per year (mas/y in the following) and 3,387.46 mas/y respectively.

The actual experimental precision allows for detecting such rates for both the LAGEOS satellites [Ciufolini, 1996]. Indeed, for the perigee the observable quantity is $r \equiv e a \dot{\omega}$; at 
present the RMS of the residuals of the best orbital fits amounts to $\delta r_{\text {exp }} \sim 1 \mathrm{~cm}$, or less, for the two LAGEOS satellites, over several orbits and with a set of force models (i.e. not including modeling errors). Since the LAGEOS eccentricity is $e_{\mathrm{I}}=4.5 \times 10^{-3}$ the accuracy in detecting the perigee is $\delta \omega_{\exp }^{\mathrm{I}} \sim \delta r_{\exp } /\left(e_{\mathrm{I}} a_{\mathrm{I}}\right) \simeq 37$ mas. So that, over 1 year the relative accuracy in the measurement of the relativistic perigee shift would be of the order of $1 \times 10^{-2}$. For LAGEOS II this measurement accuracy is better than for LAGEOS. Indeed, the LAGEOS II eccentricity is $e_{\mathrm{II}}=1.4 \times 10^{-2}$, thus $\delta \omega_{\exp }^{\mathrm{II}} \sim 12$ mas; this may yield an accuracy of the order of $3 \times 10^{-3}$ over 1 year. Over 8 years, by assuming the same fit error as before, it would amount to $\sim 4 \times 10^{-4}$. These considerations rule out the possibility of directly using the perigee of the other existing, spherical, geodetic laser-ranged satellites Etalon-1, Etalon-2, Ajisai, Stella, Westpac-1 because their eccentricities are even smaller than that of LAGEOS. On the contrary, Starlette has an eccentricity of the order of $2 \times 10^{-2}$; however, since it orbits at a lower altitude is more sensitive than the LAGEOS satellites to atmospheric drag and to Earth's zonal harmonics, so that would be difficult to process its data at an acceptable level of accuracy (See also section 4). Accordingly, in order to detect the gravitoelectric relativistic shift in the gravitational field of Earth, we will focus on the perigee of LAGEOS and especially of LAGEOS II .

\section{The systematic errors}

The perigee of an Earth satellite is a "non-clean" orbital element in the sense that it is affected by a large number of aliasing classical forces inducing systematic errors which must be carefully identified and analyzed.

\subsection{The static geopotential error}

The most important source of systematic errors in such a measurement is represented by the mismodeling of the classical perigee precession induced by the errors in even zonal harmonics of the Earth's gravitational field [Kaula, 1966]. This error is really critical because the resulting aliasing trend cannot be removed from the data and nothing can be done about it apart from assessing the related error as reliably as possible.

By using the covariance matrix of the EGM96 Earth gravity model [Lemoine et al., 1998] 
and adding the correlated terms in a root-sum-square fashion up to degree $l=20$, we obtain for LAGEOS a systematic error $\delta \nu / \nu_{\text {zonals }}=8.1 \times 10^{-3}$ whereas for LAGEOS II we have $\delta \nu / \nu_{\text {zonals }}=1.5 \times 10^{-2}$. Since the major source of error lies in the uncertainty of the first two mismodeled even zonal harmonics $\delta J_{2}$ and $\delta J_{4}$, in order to eliminate most of the static and dynamical even zonal terms of the geopotential, following the method in [Ciufolini, 1996] for the measurement of the gravitomagnetic Lense-Thirring effect, we search for suitable combinations of the orbital residuals of the existing laser-ranged satellites. In Tab.11 we report the most promising combinations: their general form is

$$
\delta \dot{\omega}^{\mathrm{II}}+\sum_{\mathrm{i}=1}^{\mathrm{N}} c_{\mathrm{i}} \delta \dot{\Omega}^{\mathrm{i}}+c_{\mathrm{N}+1} \delta \dot{\omega}^{\mathrm{I}}=x_{\mathrm{GR}} \nu,
$$

in where $N$ is the number of the nodes of different laser-ranged satellites employed, $x_{\mathrm{GR}}$ is the slope, in mas/y, of the relativistic trend to be measured, in Tab.11 $\delta \nu / \nu_{\text {zonals }}$ is the systematic error induced by the even zonal harmonics up to degree $l=20$ calculated with the EGM96 covariance matrix. The coefficients $c_{i}$ are determined in terms of the orbital parameters of the satellites entering the combinations.

Table 1: Combined residuals: numerical values

\begin{tabular}{lllllll}
\hline & $\Omega^{\mathrm{II}}$ & $\Omega^{\mathrm{I}}$ & $\Omega^{\mathrm{Aj}}$ & $\omega^{\mathrm{I}}$ & & \\
\hline$\kappa$ & $c_{1}$ & $c_{2}$ & $c_{3}$ & $c_{4}$ & $x_{\mathrm{GR}}(\mathrm{mas} / \mathrm{y})$ & $\delta \nu / \nu_{\text {zonals }}$ \\
\hline 1 & -0.87 & -2.86 & 0 & 0 & $3,387.46$ & $6.59 \times 10^{-3}$ \\
2 & -2.51 & -4.37 & 0 & 2.51 & $11,704.92$ & $1.1 \times 10^{-3}$ \\
3 & -1.96 & -3.69 & 0.037 & 1.37 & $7,928.51$ & $8.1 \times 10^{-4}$ \\
\hline
\end{tabular}

It is important to stress that the use of the LAGEOS satellites, due to their altitude, makes our measurement substantially insensitive to the errors in the zonal harmonics of degree $l>20$, so that our estimates of $\delta \nu / \nu_{\text {zonals }}$ presented here are valid even in the case that the EGM96 covariance matrix for degrees higher than $l>20$ would not be accurate enough.

In addition to LAGEOS and LAGEOS II we have only considered Ajisai [Iorio, 2002a] since it is well tracked, contrary for example to the Etalon satellites, and it would be less demanding than for the other satellites to reduce its laser-ranged data to a level of accuracy comparable to that of LAGEOS and LAGEOS II. Moreover, the other laser-ranged satellites 
orbit at lower altitudes, therefore they are more sensitive to terms of the geopotential of higher degree. Consequently, as confirmed by numerical calculations, the inclusion of their data in the combined residuals would increase $\delta \nu / \nu_{\text {zonals }} \rrbracket$.

It is important to point out that the uncertainties quoted in Tab.1 for $\delta \nu / \nu_{\text {zonals }}$ will be reduced in the near future when the new gravity models from the CHAMP and GRACE missions will be released [Pavlis, 2002a].

\subsection{The time-dependent systematic errors}

In regard to the evaluation of the impact of the other sources of systematic errors, the role of the coefficients $c_{i}$ entering the combinations has to be taken into account, indeed, according to their magnitude, they can reduce or emphasize the effects of the perturbing forces acting on the orbital elements weighted by them.

For example, for the combination 2 of Table 1, over 1 year, the impact of the error in measuring the perigee rate of LAGEOS amounts to about $2.5 \times\left(3.1 \times 10^{-3}\right)=7.7 \times 10^{-3}$ while for combination 3 it is about $1.37 \times\left(4.7 \times 10^{-3}\right)=6.4 \times 10^{-3}$.

In this paper we only consider in detail the combination 1 of Table 1

$$
\delta \dot{\omega}^{\mathrm{II}}-0.87 \times \delta \dot{\Omega}^{\mathrm{II}}-2.86 \times \delta \dot{\Omega}^{\mathrm{I}}=3,387.46 \times \nu,
$$

so that we exploit the insight acquired with the Lense-Thirring LAGEOS experiment [Ciufolini et al., 1996; 1997; 1998].

The long-period harmonic perturbations, according to their periods $P$ and to the adopted observational time span $T_{\text {obs }}$, may turn out to be less insidious than the mismodeled secular perturbations due to the zonal harmonics of the geopotential since, if $P<T_{\text {obs }}$ and $T_{\text {obs }}=$ $n P, n=1,2, \ldots$ they average out; if their periods are shorter than the time span they can be viewed as empirically fitted quantities which can be subsequently removed from the signal.

The results and estimates recently obtained for the LAGEOS gravitomagnetic experiment can be applied to our proposed measurement. Indeed, the solid Earth and ocean tidal perturbations on LAGEOS and LAGEOS II and their impact on the Lense-Thirring effect have been

\footnotetext{
${ }^{1}$ About combination 3 , it should be noted that, in order to obtain more reliable and accurate estimates of the systematic error due to the even zonal harmonics of the geopotential, the calculation of the error should include terms with degree higher than $l=20$ due to the sensitivity of Ajisai to such higher degree terms.
} 
studied in [Iorio, 2001; Iorio and Pavlis, 2001; Pavlis and Iorio, 2002], while the role played by the non-gravitational perturbations has been analyzed in [Lucchesi, 2001; 2002]. Regarding the atmospheric drag, whose impact might be thought of as a serious drawback, especially for the perigee of LAGEOS II, in [Ciufolini et al., 1997] it is shown that it essentially averages out over an orbital revolution.

In Tab.2 we summarize the results obtained with the proposed combination using LAGEOS and LAGEOS II for a 8-year time span with orbital fits, arcs, each of 7 days. In assessing the total systematic error we have accounted for the fact that the gravitational errors are not independent, indeed we have simply summed them up; then we have added these uncertainties and the other independent systematic errors in a root-sum-square fashion. In Tab.2 we can observe that the error budget is mainly dominated by the even zonal harmonics and by the LAGEOS II perigee measurement error. In regard to the non-gravitational errors, according to the results of [Lucchesi, 2001] for the direct solar radiation pressure and the Earth's albedo and to the results of [Lucchesi, 2002] for the thermal thrust perturbations and the asymmetric reflectivity, the corresponding uncertainty would amount to almost $1 \times 10^{-2}$ over 7 years. In obtaining this result a very pessimistic approach has been adopted by assuming a mismodeling of $20 \%$ for all the perturbing effects except for the direct solar radiation pressure which has been assumed to have an uncertainty at the $0.5 \%$ level. However, we stress that only the Earth's thermal thrust, or Yarkovski-Rubincam effect, induces a mismodeled linear trend whose impact would amount to about $1 \times 10^{-4}$ : the other non-gravitational forces are time-varying with known periodicities and can be fitted and removed from the signal as done for the tidal perturbations.

Table 2: Preliminary error budget: $T_{o b s}=8$ years, $\Delta t=7$ days.

\begin{tabular}{ll}
\hline Even zonal harmonics (par. 3.2) & $6.59 \times 10^{-3}$ \\
$J_{3}$ geopotential [Pavlis and Iorio, 2002] & $3.2 \times 10^{-4}$ \\
Tides [Iorio, 2001; Pavlis and Iorio, 2002] & $4.4 \times 10^{-4}$ \\
Non-gravitational effects [Lucchesi, 2001; 2002] & $1 \times 10^{-4}$ \\
Measurement error in LAGEOS II perigee (sect. 2) & $4 \times 10^{-4}$ \\
\hline Total systematic error & $7.3 \times 10^{-3}$ \\
\hline
\end{tabular}


Forthcoming improvements in the Earth gravity field modeling achievable with the CHAMP and GRACE data have been recently extensively studied and reported in [Pavlis, 2002a]. They will yield important improvements in the accuracy of our experiment. We finally observe that we already have the required laser-ranging data since, at present, we have almost 10 years of LAGEOS and LAGEOS II data.

\subsection{The parameters $\beta$ and $\gamma$}

The results obtained for the combination 1 examined here, together with $\delta \eta=8 \times 10^{-4}$ reported in [Anderson and Williams, 2001] for the combination $\eta=4 \beta-\gamma-3$ [Nordtvedt, 1968; 1991], would allow to measure $\beta=\frac{2}{7} \eta+\frac{3}{7} \nu+\frac{4}{7}$ at the level $\sim 3 \times 10^{-3}$ independently of other measurements of $\gamma$; this result, which is of the same order of magnitude of that obtained with radar ranging [Shapiro, 1990], should be compared with the most recent $\delta \beta=4.7 \times 10^{-4}$, obtained with LLR data [Anderson and Williams, 2001].

The parameter $\gamma$, that may be written as $\gamma=\frac{1}{7} \eta+\frac{12}{7} \nu-\frac{5}{7}$, would be measured less precisely: $\delta \gamma=1.2 \times 10^{-2}$. However, $\gamma$ can be directly measured via light deflection or radar time delay. For example, in [Frœschlé et al., 1997] the result $\gamma=0.997 \pm 3 \times 10^{-3}$ is based on the astrometric observations of electromagnetic waves deflection in the visible frequency. By using the Viking time delay [Reasenberg et al., 1979], $\gamma=1.000 \pm 2 \times 10^{-3}$ was obtained; the quoted uncertainty allows for possible systematic errors. A more recent measurement based on the time delay with the NEAR spacecraft [Elliott et al., 1998] claims an uncertainty $\leq 10^{-3}$.

For an updated overview of the present status of the measurements of the PPN parameters, see [Will, 2001]

\section{The role of LARES and Starlette}

The proposed LARES satellite [Ciufolini, 1998] will enable us to perform several general relativistic tests and geophysical measurements. In this section we investigate its role for the measurement of the gravitoelectric perigee advance that would be well detectable on LARES thanks to its proposed relatively large eccentricity: $e_{\mathrm{LR}} \simeq 0.04$. 
A possible observable is the following combination of orbital residuals

$$
\delta \dot{\omega}^{\mathrm{II}}+c_{1} \delta \dot{\omega}^{\mathrm{LR}}+c_{2} \delta \dot{\Omega}^{\mathrm{II}}=x_{\mathrm{GR}} \nu
$$

with

$$
\begin{aligned}
c_{1} & =-4.71 \\
c_{2} & =2.26 \\
x_{\mathrm{GR}} & =-12,117.4 \mathrm{mas} / \mathrm{y} .
\end{aligned}
$$

The coefficients $c_{i}$ depend on the orbital parameters of the selected satellites and have been calculated corresponding to the proposed orbital parameters of LARES: $a_{\mathrm{LR}}=12,270 \mathrm{~km}$, $i_{\mathrm{LR}}=70 \mathrm{deg}$ and $e_{\mathrm{LR}}=0.04$. Let us now calculate the systematic errors induced by the even zonal harmonics of the geopotential, according to the EGM96 gravity model. Eq. (雨) would allow to cancel out the errors due to the first four even zonal harmonics; the impact of the remaining ones, with degree higher than four, amounts to an uncertainty of about $6 \times 10^{-3}$. This error will further reduce when the new gravity models from the CHAMP and GRACE missions will be available. An interesting feature of eq. (4) is that the impact of the nongravitational perturbations would produce an uncertainty of about $3.9 \times 10^{-3}$ over a time span of 7 years, according to the very conservative estimates discussed above; in particular the Earth thermal thrust, i.e. the so-called Yarkovski-Rubincam effect, would induce a mismodeled secular trend with an uncertainty of only $6.4 \times 10^{-5}$. The inclusion of LARES would thus represent an improvement with respect to the LAGEOS and LAGEOS II scenario previously outlined, especially when, in the near future, the impact of the mismodeled non-gravitational perturbations will increase relatively to the gravitational perturbations thanks to the more accurate Earth's gravity field models.

Among the existing laser-ranged satellites, Starlette, with its relatively large eccentricity of 0.0204, seems to be a natural candidate for the measurement of the perigee advance. Unfortunately, it orbits at an altitude much lower than the LAGEOS satellites $\left(a_{\mathrm{Str}}=7,331 \mathrm{~km}\right)$ and thus the mismodeling of the even zonal harmonics of the geopotential would induce large systematic errors in the measurement of the perigee rate. It turns out that the inclusion of the perigee of Starlette in the combined residuals if, on the one hand, provides a further observable 
and thus would allow to cancel out the error of an additional mismodeled even zonal harmonic, on the other hand it raises the total error due to the geopotential, even with the LARES data. Indeed, in a combination including the nodes of LAGEOS and LAGEOS II and the perigees of LAGEOS II and Starlette, the relative error due to the geopotential amounts to only $6.2 \times 10^{-2}$. This rules out the possibility of using the Starlette data, at present level of knowledge of the Earth's gravitational field, to improve the measurement of the relativistic perigee advance.

\section{Conclusions}

The main features of our proposed measurement of perigee advance using laser-ranged satellites are the following:

- It will provide a test of a general relativistic effect, i.e. the pericenter advance, in the gravitational field of Earth, complementary to similar tests in the field of Sun. Indeed, such a measurement in the gravitational field of Earth would place stronger limits on possible, very weak, Yukawa-type components of the gravitational interaction with a finite range of the order of $10^{4} \mathrm{~km}$ [Iorio, 2002b].

- For a suitable combination of the orbital residuals of the nodes of LAGEOS and LAGEOS II and perigee of LAGEOS II, the relative error due to the mismodeled classical precessions by the static even zonal harmonics of the geopotential, which is the most insidious source of systematic error, amounts, according to the EGM96 Earth gravity model, to about $6 \times 10^{-3}$. The total error in our proposed combination of orbital elements, also due to the other time-dependent systematic errors, amounts, over an observational time span of eight years, to almost $7 \times 10^{-3}$.

- In the near future the knowledge of the Earth gravitational field will increase thanks to the data from the CHAMP and GRACE missions. Moreover, the rms accuracy in measuring the position of the LAGEOS satellites should reach the few $\mathrm{mm}$ level. Consequently, there will be an increase in the accuracy of our measurement.

- In regard to the terrestrial space environment, which induces a large number of orbital perturbations on the satellites, it is very well studied and the action of these perturbations, 
especially on the LAGEOS satellites, is very well modeled. The data processing would be reliably based on the large experience collected in several years of analysis of the LAGEOS orbits [Pavlis, 2002b]. Moreover, it would be relatively fast and easy to include the future improvements in the force modeling in our analysis, e.g. the improvements due to a better experimental and theoretical knowledge of the rotation rate and spin axis orientation of LAGEOS type satellites.

- The proposed measurement, as an additional outcome, from $\frac{2+2 \gamma-\beta}{3}$ in conjunction with $\eta=4 \beta-\gamma-3$ from Lunar Laser Ranging, would allow to obtain the PPN parameters $\gamma$ and $\beta$ in the gravitational field of Earth (see above) with an accuracy at the level of $10^{-2}-10^{-3}$. This accuracy would improve with the new Earth's gravitational models from CHAMP and GRACE.

- Starlette data, although this satellite has a relatively eccentric orbit, should not be included in the combined residuals. Indeed, Starlette would raise the systematic error in the geopotential due to its lower altitude with respect to the LAGEOS satellites and thus due to its higher sensitivity to the even zonal harmonics of the terrestrial gravitational field.

- The use of the perigee of the proposed LARES satellite, for a suitable combination of observables including also the node and the perigee of LAGEOS II, would allow to reduce the systematic relative error due to the mismodeled even zonal harmonics of the geopotential to about $6 \times 10^{-3}$, according to EGM96. This proposed measurement would also take advantage of the new Earth gravity models from CHAMP and GRACE. According to conservative estimates, the impact of the non-gravitational perturbations would amount to about $4 \times 10^{-3}$ over 7 years; in particular the impact of the mismodeled Earth thermal thrust, or Yarkovski-Rubincam effect, would amount to only about $6 \times 10^{-5}$.

\section{Acknowledgements}

L. I. would like to thank L. Guerriero for his encouragement and support, C.M. Will and I.I. Shapiro for the useful information kindly supplied to him. E. C. Pavlis gratefully acknowledges 
NIMA support through the NURI grant NMA201-01-1-2008.

\section{References}

[Anderson and Williams, 2001] Anderson, J.D., and J.G. Williams, Long-range tests of the equivalence principle, Class. and Quantum Grav., 18, 2447-2456, 2001.

[Balogh et al., 2000] Balogh, et al., BepiColombo An Interdisciplinary Cornerstone Mission to the Planet Mercury, ESA-SCI, 1, pp. 21-24 and pp. 35-38, 2000.

[Ciufolini and Matzner, 1992] Ciufolini, I., and R. Matzner, Non-Riemannian theories of gravity and lunar and satellite laser ranging, Int. J. of Mod. Phys. A, 7, 4, 843-852, 1992.

[Ciufolini and Wheeler, 1995] Ciufolini, I., and J. A. Wheeler, Gravitation and Inertia, 498 pp., Princeton University Press, New York, 1995.

[Ciufolini, 1996] Ciufolini, I., On a new method to measure the gravitomagnetic field using two orbiting satellites, Il Nuovo Cimento, 109A, 12, 1709-1720, 1996.

[Ciufolini et al., 1996] Ciufolini I., D. Lucchesi, F. Vespe and A. Mandiello, Measurement of dragging of inertial frames and gravitomagnetic field using laser-ranged satellites, $I l$ Nuovo Cimento 109A, 5, 575-590, 1996.

[Ciufolini et al., 1997] Ciufolini, I., F. Chieppa, D. Lucchesi, and F. Vespe, Test of LenseThirring orbital shift due to spin, Class. and Quantum Grav., 14, 2701-2726, 1997.

[Ciufolini et al., 1998] Ciufolini, I., E. Pavlis, F. Chieppa, E. Fernandes-Vieira, and J. PérezMercader, Test of General Relativity and Measurement of the Lense-Thirring Effect with Two Earth Satellites, Science, 279, 2100-2103, 1998.

[Ciufolini, 1998] Ciufolini, I., Accurate Test of the Relativistic Perigee Precession and Measurement of the Relativistic Parameters $\beta$ and $\gamma$ using LARES, in LARES Phase-A Study, pp. 39-40, Rome, 1998. 
[Elliott et al., 1998] Elliott, A. A., R. W. Hellings, and J. K. Miller, PPN Gamma Results from the NEAR Conjunction, AAS 191st Meeting, Washington, DC, Session 48, no. 09, January 1998.

[Frœeschlé et al., 1997] Froschlé, M., F. Mignard, and F. Arenou, Determination of the PPN parameter $\gamma$ with the Hipparcos data, ESA SP-402, 1997.

[Iorio, 2001] Iorio, L., Earth tides and Lense-Thirring effect, Celest. Mech., 79 201-230, 2001.

[Iorio, 2002a] Iorio, L., Testing General Relativity with LAGEOS, LAGEOS II and Ajisai laserranged satellites, J. of the Geodetic Soc. of Japan, 48 (1), 13-20, 2002a.

[Iorio, 2002b] Iorio, L., Constraints to a Yukawa gravitational potential from laser data to LAGEOS satellites, Physics Lett. A, 298(5-6), 315-318, 2002 b.

[Iorio and Pavlis, 2001] Iorio, L., and E. C. Pavlis, Tidal satellite perturbations and the LenseThirring effect, J. of the Geodetic Soc. of Japan, 47, 169-173, 2001.

[Kaula, 1966] Kaula, W. M., Theory of Satellite Geodesy, 124 pp., Blaisdell Publishing Company, Waltham, 1966.

[Lemoine et al., 1998] Lemoine, F. G., et al., The Development of the Joint NASA GSFC and the National Imagery Mapping Agency (NIMA) Geopotential Model EGM96, NASA/TP-1998-206861, 1998.

[Lucchesi, 2001] Lucchesi, D., Reassessment of the error modelling of non-gravitational perturbations on LAGEOS II and their impact in the Lense-Thirring determination. Part I, Plan. and Space Sci., 49, 447-463, 2001.

[Lucchesi, 2002] Lucchesi, D., Reassessment of the error modelling of non-gravitational perturbations on LAGEOS II and their impact in the Lense-Thirring determination. Part II. To appear in Plan. and Space Sci., 2002.

[Milani et al., 2001a] Milani, A., A. Rossi, D. Vokrouhlický, D. Villani, and C. Bonanno, Gravity field and rotation state of Mercury from the BepiColombo Radio Science Experiment, Plan. and Space Sci., 49, 1579-1596, 2001. 
[Milani et al., 2001b] Milani, A., D. Vokrouhlický, D. Villani, C. Bonanno, and A. Rossi, Testing general relativity with the BepiColombo radio science experiment, Quaderni del Dipartimento di Matematica, Università di Pisa, 4.89.1348, 2001.

[Misner et al., 1973] C. W. Misner, K. S. Thorne and J. A. Wheeler, Gravitation, 1279 pp., Freeman, San Francisco, 1973.

$[N i, 2001] \mathrm{Ni}$, Wei-Tou, Testing Relativistic Gravity and Measuring Solar System Parameters via Optical Space Missions, in: Lämmerzahl, C., C.W.F. Everitt, and F.W. Hehl (Eds.), Gyros, Clocks, Interferometers...:Testing Relativistic Gravity in Space, Lecture Note in Physics 562, 507 pp., Springer Verlag, Berlin, 2001.

[Nordvedt, 1968] Nordvedt, K., Testing Relativity with Laser Ranging to the Moon, Phys. Rev., 170, 1186-1187, 1968.

[Nordvedt, 1991] Nordvedt, K., Lunar laser ranging reexamined. The non-null relativistic contribution, Phys. Rev. D, 43, 3131-3135, 1991.

[Pavlis, 2002a] Pavlis, E. C., Geodetic Contributions to Gravitational Experiments in Space, in: Cianci, R.(Ed.), Recent Developments in General Relativity, Springer Verlag, Milan, 2002.

[Pavlis, 2002b] Pavlis, E. C., Dynamic Determination of Origin and Scale the Earth System from Satellite Laser Ranging, in: Proceedings of the 2001 IAG Scientific Assembly: Vistas for Geodesy in the New Millennium, the International Association of Geodesy, Budapest, Hungary, September 2-7, 2001, electronic publication (CD), 2002.

[Pavlis and Iorio, 2002] Pavlis, E. C., and L. Iorio, The impact of tidal errors on the determination of the Lense-Thirring effect from satellite laser ranging, Int. J. of Mod. Phys. D, 11(4), 599-618, 2002.

[Pitjeva, 1993] Pitjeva, E.V., Experimental testing of relativistic effects, variability of the gravitational constant and topography of Mercury surface from radar observations 19641989, Celest. Mech., 55, 313-321, 1993. 
[Reasenberg, 1979] Reasenberg, R. D., Viking Relativity Experiment: Verification of Signal Retardation by Solar Gravity, Astrophys. J., 234, 124-B5, L219-L221, 1979.

[Shapiro et al., 1972] Shapiro, I. et al., Mercury' s Perihelion Advance: Determination By Radar, Phys Rev. Lett., 28, 1594-1597, 1972.

[Shapiro et al., 1976] Shapiro, I., C. Counselman, and R. King, Verification of the Principle of Equivalence for Massive Bodies, Phys Rev. Lett., 36, 555-558, 1976.

[Shapiro, 1990] Shapiro, I., Solar system tests of general relativity: recent results and present plans, in Proceedings of the 12th International Conference on General Relativity and Gravitation, University of Colorado at Boulder, 1989, edited by N. Ashby, D. Bartlett, and W. Wyss, Cambridge University Press, Cambridge, 313-330, 1990.

[Will, 1993] Will, C M., Theory and Experiment in Gravitational Physics, 2nd edition, Cambridge University Press, Cambridge, 1993.

[Will, 2001] Will, C M., The Confrontation between General Relativity and Experiment, Living Rev. Relativity 2001-4 (January, 2001), [Article in Online Journal] cited on: 09 Aug 2001, http://www.livingreviews.org/Articles/Volume4/2001-4will 